\title{
Predicting outcome in severe traumatic brain injury using a simple prognostic model
}

\author{
S Sobuwa, ${ }^{1}$ MSc (Med) (Emerg Med); H B Hartzenberg, ${ }^{2}$ MMed (Neurosurg); H Geduld, ${ }^{3}$ MMed (Emerg Med); C Uys, ${ }^{4}$ MSc (Stats) \\ ${ }^{1}$ Department of Emergency Medical Sciences, Faculty of Health and Wellness Sciences, Cape Peninsula University of Technology, \\ Cape Town, South Africa \\ ${ }^{2}$ Division of Neurosurgery, Stellenbosch University, Parow, South Africa \\ ${ }^{3}$ Division of Emergency Medicine, University of Cape Town, South Africa \\ ${ }^{4}$ Centre for Postgraduate Studies, Cape Peninsula University of Technology, Cape Town, South Africa
}

Correspondingauthor: S Sobuwa (simpiwesobuwa@gmail.com)

Background. Several studies have made it possible to predict outcome in severe traumatic brain injury (TBI) making it beneficial as an aid for clinical decision-making in the emergency setting. However, reliable predictive models are lacking for resource-limited prehospital settings such as those in developing countries like South Africa.

Objective. To develop a simple predictive model for severe TBI using clinical variables in a South African prehospital setting.

Methods. All consecutive patients admitted at two level-one centres in Cape Town, South Africa, for severe TBI were included. A binary logistic regression model was used, which included three predictor variables: oxygen saturation $\left(\mathrm{SpO}_{2}\right)$, Glasgow Coma Scale $(\mathrm{GCS})$ and pupil reactivity. The Glasgow Outcome Scale was used to assess outcome on hospital discharge.

Results. A total of $74.4 \%$ of the outcomes were correctly predicted by the logistic regression model. The model demonstrated $\mathrm{SpO}_{2}$ $(p=0.019)$, GCS $(p=0.001)$ and pupil reactivity $(p=0.002)$ as independently significant predictors of outcome in severe TBI. Odds ratios of a good outcome were $3.148\left(\mathrm{SpO}_{2} \geq 90 \%\right), 5.108$ (GCS 6 - 8) and 4.405 (pupils bilaterally reactive).

Conclusion. This model is potentially useful for effective predictions of outcome in severe TBI.

S Afr Med J 2014;104(7):492-494. DOI:10.7196/SAMJ.7720

Several studies ${ }^{[1-4]}$ have made it possible to predict outcome in severe traumatic brain injury (TBI) making it beneficial as an aid for clinical decision-making in the emergency setting. Accurate assessment of prognosis is crucial in multi-casualty incidents so that prehospital emergency care practitioners can focus their attention on patients predicted to have a good outcome. No single prognostic model is practised broadly despite many models being described in the literature. Most models are derived from developed nations, which potentially offer superior intensive healthcare services 
owing to their better resources, resulting in outcomes of TBI that are different to those of developing countries. ${ }^{[5]} \mathrm{A}$ model that will be used in the prehospital setting should include variables that can be measured instantly and easily with resources available to prehospital emergency care providers.

We aimed to develop a simple predictive model for severe TBI using clinical variables.

\section{Methods}

All consecutive patients with severe TBI in the Cape Town Metropole from 1 January 2009 to 31 August 2011 admitted to Groote Schuur Hospital (GSH) or Tygerberg Hospital (TBH) were enrolled in the study. The patients were enrolled if they were $\geq 16$ years of age. Patients who were transferred to TBH and GSH from another facility were excluded from the study, as were those who had sustained penetrating head trauma. Patients who were declared dead on scene were not eligible for the study.

The Glasgow Outcome Scale (GOS) (Table 1) was used to assess outcome. For the purposes of this study, outcome was divided into good (good recovery or moderate disability) and poor (dead, vegetative state or severe disability). Initially the model included five predictor variables for the two outcomes: $(i)$ oxygen saturation $\left(\mathrm{SpO}_{2}\right)(\geq 90 \%$ and $<90 \%)$; (ii) Glasgow Coma Scale (GCS) (3 - 5 and $6-8$ ) measured on arrival at the scene; (iii) pupil reactivity (pupils bilaterally reactive and all other findings); (iv) systolic blood pressure (SBP) ( $>90 \mathrm{mmHg}$ and $<90 \mathrm{mmHg}$ ); and $(v)$ presence of a concomitant injury or not. Presence of a concomitant injury and SBP did not have a significant impact on the model and were omitted, leaving three variables $-\mathrm{SpO}_{2}$, GCS and pupil reactivity.

The predictor variables were therefore reclassified into binary variables. A binary logistic regression model was fitted using the three predictor variables as categorical independent binary predictors and the GOS as the dependent binary variable. Binary logistic regression predicts binary outcomes (i.e. poor or good in the case of this study) of the dependent variable using a number of independent predictor variables. The goodness-of-fit of this model is measured by the Hosmer-Lemeshow $\chi^{2}$ statistic, which gives a non-significant result $\left(\chi^{2}=2.986\right.$; degrees of freedom $\left.7 ; p=0.622\right)$. The Hosmer-Lemeshow statistic tests the null hypothesis that there is a linear relationship between the independent predictors and the logarithmic odds of the dependent binary variable. The hypothesis is tested using a $\chi^{2}$ distribution and a non-significant result is an indication of a good fit.

Estimated $\beta$-values were coefficients in the logistic regression model used to calculate the odds ratio (OR) and the subsequent probability of a good outcome as follows:

OR $($ good outcome $)=\mathrm{e}^{-2.717+1.673 \times \mathrm{GCS}+1.187 \times \mathrm{SpO}_{2}+1.564 \times \text { pupil reactivity }}$

Probability $($ good outcome $)=\frac{\mathrm{OR}}{1+\mathrm{OR}}$

\section{Results}

A total of 124 patients were enrolled during the period 1 January 2009 - 31 August 2011. There was an equal split of patients $(n=62)$ from GSH and from TBH. There was no significant difference in outcome between the two hospitals $(p=0.583)$. Males comprised $89 \%$ of the sample population, with road traffic accidents being the leading cause of injury. The mortality rate for the study population was $39 \%$, with $60 \%$ having a good outcome.

The binary logistic regression model correctly predicted $74.4 \%$ of the patients to be in one of the two outcome groups (Table 2).

With our model, bilateral pupil reactivity, GCS of $6-8$ and $\mathrm{SpO}_{2}$ $\geq 90 \%$ were shown to predict a positive outcome following severe TBI. The higher the GCS score, the better the outcome. Increasing age was not associated with poor outcome in this analysis. Having bilateral reactive pupils increased the odds of a good outcome by $340.5 \%$.

The odds of a good outcome increased by $214.8 \%$ in patients with $\mathrm{SpO}_{2} \geq 90 \%$. The presence of hypotension did not have a significant effect on the model.

ORs and probabilities for different scenarios are summarised in Table 3. The odds of the patient having a good outcome on the GOS was 5.5124:1 and the probability of a good outcome was 0.8464 if the patient had a GCS of $6-8, \mathrm{SpO}_{2}$ of $>90 \%$ with bilateral reactive pupils. It was evident that when there were $\geq 2$ negative predictor values for a patient, the OR dropped significantly to $<1$ (Table 3 , scenario 5).

\section{Discussion}

Using our logistic regression model, bilateral pupil reactivity, GCS of 6 - 8 and $\mathrm{SpO}_{2} \geq 90 \%$ were shown to predict a positive outcome following severe TBI. These variables have been previously reported to predict prognosis in TBI. ${ }^{[1-4,6]}$ The initial GCS on scene appears to be the most sensitive of these variables as seen in the model. Our analysis suggests that the higher the GCS score, the better the outcome. In fact, the OR suggests that having a GCS score of $6-8$ in the case of severe TBI increases the odds of a good outcome by $410.8 \%$. Contrary to other studies that found increasing age to be associated with adverse outcome, ${ }^{[3,7]}$ increasing age was not associated with poor outcome in this analysis. This could be due to the fact that patients $<16$ years of age were not included in the study. In the CRASH trial, a poorer outcome

\begin{tabular}{ll} 
Table 1. Glasgow Outcome Scale & \\
\hline Level & Term \\
\hline 1 & Dead \\
2 & Persistent vegetative state \\
3 & Severe disability \\
4 & Moderate disability \\
5 & Good recovery
\end{tabular}

Table 2. Logistic regression analysis*

\begin{tabular}{|c|c|c|c|c|c|c|}
\hline & $\beta$-value & SE & Wald $\chi^{2}$ & df & $p$-value & OR \\
\hline GCS $(6-8)$ & 1.673 & \pm 0.446 & 14.032 & 1 & 0.000 & 5.108 \\
\hline $\mathrm{SpO}_{2}(>90 \%)$ & 1.187 & \pm 0.506 & 5.515 & 1 & 0.019 & 3.148 \\
\hline Pupil reactivity (bilateral) & 1.564 & \pm 0.513 & 9.303 & 1 & 0.002 & 4.405 \\
\hline Constant & -2.717 & \pm 0.560 & 23.574 & 1 & 0.000 & 15.133 \\
\hline
\end{tabular}


Table 3. Odds ratios and probability of good outcome for various situations

\begin{tabular}{lllllll}
\hline Scenario & GCS & $\mathrm{SpO}_{2}(\%)$ & Pupil reactivity & OR & Probability & Clinical PPV, $\boldsymbol{n}$ \\
\hline 8 & $6-8$ & $\geq 90$ & Bilaterally reactive & 5.5124 & 0.8464 & 3 \\
6 & $6-8$ & $<90$ & Bilaterally reactive & 1.6820 & 0.6271 & 2 \\
7 & $\geq 90$ & All other findings & 1.1537 & 0.5357 & 2 & 2 \\
4 & $3-8$ & Bilaterally reactive & 1.0346 & 0.5085 & 1 \\
5 & $3-5$ & All other findings & 0.3520 & 0.2604 & 1 \\
2 & $6-8$ & $<90$ & Bilaterally reactive & 0.3157 & 0.2399 & 1 \\
3 & $3-5$ & $<90$ & All other findings & 0.2165 & 0.1780 & 0 \\
1 & $3-5$ & $\geq 90$ & All other findings & 0.0661 & 0.0620 &
\end{tabular}

was present after the age of 40 years, ${ }^{[3]}$ suggesting that there is a major difference between patients $>40$ years and those $<40$ years.

There is no published literature in the prehospital setting investigating the association between pupillary findings and outcome following severe TBI. However, some authors have suggested a strong relationship between pupillary findings and outcome in the in-hospital setting. ${ }^{[1,8,9]}$ The pupillary light reflex is an indirect measure of brain stem injury and herniation as compression of the third cranial nerve results in a fixed and dilated pupil. Jiang et al..$^{[10]}$ and Signorini et al. ${ }^{[4]}$ discovered a strong relationship between fixed, dilated pupils and ultimate mortality.

Normally, an ipsilateral fixed and dilated pupil suggests lateral transtentorial herniation, while bilaterally fixed and dilated pupils are consistent with central transtentorial herniation in a fully resuscitated patient. In our analysis, having bilateral reactive pupils increased the odds of a good outcome by $340.5 \%$.

The increased odds of a good outcome (214.8\%) in patients with $\mathrm{SpO}_{2} \geq 90 \%$ emphasises the importance of airway maintenance, oxygenation and ventilation in the prehospital setting to optimise outcome for the obtunded patient with severe TBI. However, the Brain Trauma Foundation does not recommend intubation of ground transport patients where the $\mathrm{SpO}_{2}$ is $\geq 90 \%{ }^{[11]}$ In our previous work, prehospital intubation did not demonstrate improved outcomes over basic airway management. ${ }^{[12]}$ Although it might be assumed that patients who suffer concomitant injuries would have worse outcomes, this was not shown in our analysis. The presence of hypotension did not have a significant effect on the model despite being widely reported as one the most serious influences in the TBI patient. ${ }^{[6]}$ This could be attributed to the few patients $(n=7)$ who were hypotensive in our sample population. Significant decreases in the cerebral perfusion pressure result in brain tissue ischaemia and failure of auto-regulation. The brain then becomes dependent on the mean arterial pressure for perfusion, making maintenance of cerebral perfusion and oxygenation crucial in the setting of TBI as the brain is so vulnerable to ischaemic injury.

\section{Conclusion}

This was the first simple model developed with the aim of predicting outcome in severe TBI in the South African prehospital setting. Our model was reasonably precise in correctly predicting $74.4 \%$ of the patient outcomes. It is, however, unclear what the long-term survival would have been since patients were followed up to discharge from hospital only. Future research is needed to determine how this model would impact management and outcome of severe TBI in the prehospital setting.

Author contributions. SS and CU wrote the manuscript while $\mathrm{HBH}$ and HG were responsible for the study design and critical revision of the manuscript. $\mathrm{CU}$ was also responsible for the statistical input.

References

1. Narayan RK, Greenberg RP, Miller JD, et al. Improved confidence of outcome prediction in severe head injury. J Neurosurg 1981;54(6):751-762. [http://dx.doi.org/10.3171/jns.1981.54.6.0751]

2. Mamelak AN, Pitts LH, Damron S. Predicting survival from head trauma 24 hours after injury: A practical method with therapeutic implications. J Trauma 1996;41(1):91-99. [http://dx.doi.org/10.1097/00005373199607000-00014]

3. MRC Crash Trial Collaborators. Predicting outcome after traumatic brain injury: Practical prognostic models based on large cohort of international patients. BMJ 2008;336:425. [http://dx.doi.org/10.1136/ bmj.39461.643438.25]

4. Signorini DF, Andrews PJD, Jones PA, Wardlaw JM, Miller JD. Predicting survival using simple clinical variables: A case study in traumatic brain injury. J Neurol Neurosurg Psychiatry 1999;66:20-25. [http:// dx.doi.org/10.1136/jnnp.66.1.20]

5. De Silva MI, Roberts I, Perel P, Edwards P, et al, on behalf of the CRASH Trial Collaborators. Patient outcome after traumatic brain injury in high-, middle- and low-income countries: Analysis of data on 8927 patients in 46 countries. Int J Epid 2009;38(2):452-458. [http://dx.doi.org/10.1093/ije/dyn189]

6. Chesnut RM, Marshall LF, Klauber MR, et al. The role of secondary brain injury in determining outcome from severe head injury. J Trauma 1993;34(2):216-222. [http://dx.doi.org/10.1097/00005373-19930200000006]

7. Chesnut RM, Ghajar J, Maas AI, et al. Early indicators of prognosis in severe traumatic brain injury. Neurotrauma 2000;17:557-627.

Rivas JJ, Lobato RD, Sarabia R, et al. Extradural haematoma: Analysis of factors influencing the courses of 161 patients. J Neurosurg 1988;23(1):44-51. [http://dx.doi.org/10.1227/00006123-198807000-00010]

9. Braakman R, Gelpke G, Habbema J, Maas AIR, Minderhoud JM. Systemic selection of prognostic features in patients with severe head injury. J Neurosurg 1980;6(4):362-370. [http://dx.doi.org/10.1097/00006123198004000-00002]

10. Jiang J-Y, Gao G-Y, Li W-P, Yu M-K, Zhu C. Early indicators of prognosis in 846 cases of severe traumatic brain injury. J Neurotrauma 2002;19(7):869-874. [http://dx.doi.org/10.1089/08977150260190456]

11. Badjatia N, Carney N, Crocco TJ, et al. Guidelines for prehospital management of traumatic brain injury 2nd Badjatia N, Carney N, Crocco 1], et al. Guidelines for prehospital management of traumatic brain injury
edition. Prehosp Emerg Care 2008;12(Suppl 1):S1-S52. [http://dx.doi.org/10.1080/10903120701732052]

12. Sobuwa S, Hartzenberg HB, Geduld H, Uys C. Outcomes following prehospital airway management in Sobuwa S, Hartzenberg HB, Geduld H, Uys C. Outcomes following prehospital airway management in
severe traumatic brain injury. S Afr Med J 2013;103(9):644-646. [http://dx.doi.org/10.7196/SAMJ.7035]

Accepted 10 February 2014. 